\title{
A Note on a Kinetic Model for Rod-Like Particle Suspensions
}

\author{
Xiaolong Li \\ School of Sciences, Beijing University of Posts and Telecommunications, Beijing, China \\ Email: ttlixiaolong@gmail.com
}

Received August 9, 2013; revised September 8, 2013; accepted September 23, 2013

Copyright (C) 2013 Xiaolong Li. This is an open access article distributed under the Creative Commons Attribution License, which permits unrestricted use, distribution, and reproduction in any medium, provided the original work is properly cited.

\section{ABSTRACT}

A system, coupled by an incompressible Navier-Stokes and a Fokker-Planck equation, is investigated. The global weak solution with small initial data is obtained.

Keywords: Fokker-Planck Equation; Navier-Stokes Equation; Small Initial Data

\section{Introduction}

The dilute suspensions of passive rod-like particles can be effectively modeled by a coupled microscopic Fokker-Planck equation and macroscopic Navier-Stokes equation, known as Doi model (see Doi [1]). We refer to [2] for the Doi model for suspensions of active rod-like particles without considering the effects of gravity. Recently an extended model under gravity was introduced by Hezel, Otto and Tzavaras [3], which reads

$$
\begin{gathered}
\partial_{t} f+\nabla_{x} \cdot(u f)-\Delta_{n} f+\nabla_{n} \cdot\left[(I d-n \otimes n) \nabla_{x} u n f\right] \\
=\nabla_{x} \cdot(I d+n \otimes n)\left(e_{2} f+\gamma \nabla_{x} f\right) \\
\sigma=\int_{S^{d-1}}[(d n \otimes n-I d) f] \mathrm{d} n \\
\operatorname{Re}\left[\partial_{t} u+\left(u \cdot \nabla_{x}\right) u\right]-\Delta_{x} u+\nabla_{x} p \\
=\beta \gamma \nabla_{x} \cdot \sigma-\beta\left(\int_{S^{d-1}} \mathrm{fd} n\right) e_{2} \\
\nabla_{x} \cdot u=0
\end{gathered}
$$

where $(t, x, n) \in[0, \infty) \times \Omega \times S^{d-1}, \Omega \in R^{d}$ is a bounded domain with $\partial \Omega$ of class $C^{1}$ and $S^{d-1} \subset R^{d}$ being the unit sphere; $\sigma$ is a stress tensor, $p$ is the pressure, $e_{2}$ is the unit vector in the upward direction; $\nabla_{n}$. and $\Delta_{n}$ denote the tangential divergence and Laplace-Beltrami operator on $S^{d-1}$, respectively. In this model, $f(t, x, n)$ is a distribution function which represents the configuration of a suspension of rod-like particles and $u(t, x)$ is the fluid velocity induced by the other particles in the suspension. $\operatorname{Re} \geq 0$ is a Reynolds number. The coefficients $\beta>0$ and $\gamma>0$ are constants (see [3], Remark 2.1 - 2.2).

If $\mathrm{Re}=0$, the model includes a Stokes equation. In this case, Chen, Li and Liu [4] obtain the global weak solution and its uniqueness to the two dimensional $(d=2)$ initial-boundary problem. In Remark 3.2 of [4], they point out that it is a mathematically interesting question to ask if the above result is still valid when the Stokes equation is replaced by the Navier-Stokes equation $(\operatorname{Re}>0)$, and there are some technical difficulties in solving this problem. The main purpose of this note is to answer this question by using an assumption of small initial data. See [5-7] etc. for more results on Doi related model without considering the effects of gravity.

For conciseness in presentation, we set $\operatorname{Re}=\beta=\gamma=1$ in the rest of this paper. Define

$$
\begin{aligned}
& H=\left\{u \in L^{2}(\Omega): \nabla_{x} \cdot u=0,\left.u \cdot v\right|_{\partial \Omega}=0\right\} \\
& V=\left\{u \in H_{0}^{1}(\Omega): \nabla_{x} \cdot u=0\right\}, S:=S^{1} \text { and } \\
& F(s):=s(\log s-1)+1, s \in[0, \infty) \text {. Let } L>1, \text { define }
\end{aligned}
$$
the cut-off function

$$
E^{L}:=\left\{\begin{array}{l}
0, \text { if } s \leq 0, \\
s, \text { if } s \leq L, \\
L, \text { if } s \geq L .
\end{array}\right.
$$

Set the initial and boundary conditions as follows,

$$
\begin{gathered}
\left.f\right|_{t=0}=f_{0} ;\left.u\right|_{t=0}=u_{0} ; \\
\left.(I d+n \otimes n)\left(e_{2} f+\nabla_{x} f\right) \cdot v\right|_{\partial \Omega}=0 ;\left.\quad u\right|_{\partial \Omega}=0 .
\end{gathered}
$$




\section{The Main Result}

Theorem 2.1 Let $d=2$. Suppose that $u_{0} \in H$, $f_{0} \in L^{2}(\Omega \times S)$, and $f_{0} \geq 0$ a.e. are on $\Omega \times S$. Then there exists $\varepsilon>0$, such that if

$$
\left\|u_{0}\right\|_{L^{2}(\Omega)}^{2}+\int_{\Omega \times S} F\left(f_{0}\right) \mathrm{d} n \mathrm{~d} x \leq \varepsilon,
$$

the initial-boundary problem (1)-(6) has a global weak solution $(u, f)$ which satisfies for a.e. $t \in[0, \infty)$,

$$
\begin{aligned}
& \|u(t)\|_{L^{2}(\Omega)}^{2}+2 \int_{\Omega \times S} F(f(t)) \mathrm{d} n \mathrm{~d} x+2 \int_{0}^{t}\left\|\nabla_{x} u(s)\right\|_{L^{2}(\Omega)}^{2} \mathrm{~d} s \\
& +4 \int_{0}^{t}\left(\left\|\nabla_{x} \sqrt{f(s)}\right\|_{L^{2}(\Omega \times S)}^{2}+\left\|\nabla_{n} \sqrt{f(s)}\right\|_{L^{2}(\Omega \times S)}^{2}\right) \mathrm{d} s \\
& \leq\left\|u_{0}\right\|_{L^{2}(\Omega)}^{2}+2 \int_{\Omega \times S} F\left(f_{0}\right) \mathrm{d} n \mathrm{~d} x+C\left\|f_{0}\right\|_{L^{1}(\Omega \times S)}^{2} .
\end{aligned}
$$

Definition 2.2 The weak solution $(u, f)$ is in the following sense,

$$
\begin{aligned}
& u \in L^{\infty}(0, \infty ; H) \cap L^{2}(0, \infty ; V), u \in H_{l o c}^{1}\left(0, \infty ; V^{\prime}\right) \\
& f \geq 0 \text { a.e. on }[0, \infty) \times \Omega \times S, f \in L^{\infty}\left(0, \infty ; L^{1}(\Omega \times S)\right) \\
& \nabla_{x} \sqrt{f}, \nabla_{n} \sqrt{f} \in L^{2}\left(0, \infty ; L^{2}(\Omega \times S)\right) \\
& f \in L_{l o c}^{\infty}\left(0, \infty ; L^{2}(\Omega \times S)\right) \cap L_{l o c}^{2}\left(0, \infty ; H^{1}(\Omega \times S)\right), \\
& f \in H_{l o c}^{1}\left(0, \infty ;\left(H^{3}(\Omega \times S)\right)^{\prime}\right)
\end{aligned}
$$

for any $v \in C_{0}^{\infty}([0, \infty) \times \Omega)$ with $\nabla_{x} \cdot v=0$.

$-\int_{0}^{\infty} \int_{\Omega} u \cdot \partial_{t} v \mathrm{~d} x \mathrm{~d} t+\int_{0}^{\infty} \int_{\Omega}\left(u \cdot \nabla_{x} u\right) \cdot v \mathrm{~d} x \mathrm{~d} t$

$+\int_{0}^{\infty} \int_{\Omega} \nabla_{x} u: \nabla_{x} v \mathrm{~d} x \mathrm{~d} t$

$=-\int_{0}^{\infty} \int_{\Omega \times S}(2 n \otimes n-I d) f: \nabla_{x} v \mathrm{~d} n \mathrm{~d} x \mathrm{~d} t$

$-\int_{0}^{\infty} \int_{\Omega \times S} f e_{2} \cdot v \mathrm{~d} n \mathrm{~d} x \mathrm{~d} t+\int_{\Omega} u_{0}(x) \cdot v(0, x) \mathrm{d} x ;$

for any $\varphi \in C_{0}^{\infty}([0, \infty) \times \bar{\Omega} \times S)$,

$-\int_{0}^{\infty} \int_{\Omega \times S} f \partial_{t} \varphi \mathrm{d} n \mathrm{~d} x \mathrm{~d} t-\int_{0}^{\infty} \int_{\Omega \times S}(u f) \cdot \nabla_{x} \varphi \mathrm{d} n \mathrm{~d} x \mathrm{~d} t$

$+\int_{0}^{\infty} \int_{\Omega \times S} \nabla_{n} f \cdot \nabla_{n} \varphi \mathrm{d} n \mathrm{~d} x \mathrm{~d} t$

$=\int_{0}^{\infty} \int_{\Omega \times S}\left[(I d-n \otimes n) \nabla_{x} u n f\right] \cdot \nabla_{n} \varphi \mathrm{d} n \mathrm{~d} x \mathrm{~d} t$

$-\int_{0}^{\infty} \int_{\Omega \times S}(I d+n \otimes n)\left(e_{2} f+\nabla_{x} f\right) \cdot \nabla_{x} \varphi \mathrm{d} n \mathrm{~d} x \mathrm{~d} t$

$+\int_{\Omega \times S} f_{0}(x, n) \varphi(0, x, n) \mathrm{d} n \mathrm{~d} x$.

Proof. The proof follows that of [4] (some ideas and techniques come from [8]). Here we only show the different details.

Step 1. Approximate problem. For any fixed $0<\tau \ll 1$ and for any $k \in N$, given $\left(u^{k-1}, f^{k-1}\right)$, the approximate problem with cut-off reads

$$
\begin{aligned}
& \int_{\Omega} \frac{u^{k}-u^{k-1}}{\tau} \cdot v \mathrm{~d} x+\int_{\Omega} \nabla_{x} u^{k}: \nabla_{x} v \mathrm{~d} x \\
& +\int_{\Omega}\left(u^{k-1} \cdot \nabla_{x}\right) u^{k} \cdot v \mathrm{~d} x \\
& =-\int_{\Omega \times S}(2 n \otimes n-I \mathrm{~d}) f^{k}: \nabla_{x} v \mathrm{~d} n \mathrm{~d} x \\
& -\int_{\Omega \times S} f^{k} e_{2} \cdot v \mathrm{~d} n \mathrm{~d} x, \forall v \in V ;
\end{aligned}
$$

$$
\begin{aligned}
& \int_{\Omega \times S} \frac{f^{k}-f^{k-1}}{\tau} \varphi \mathrm{d} n \mathrm{~d} x \\
& -\int_{\Omega \times S}\left(u^{k} f^{k}\right) \cdot \nabla_{x} \varphi \mathrm{d} n \mathrm{~d} x \\
& +\int_{\Omega \times S} \nabla_{n} f^{k} \cdot \nabla_{n} \varphi \mathrm{d} n \mathrm{~d} x \\
& =\int_{\Omega \times S}\left[(I d-n \otimes n) \nabla_{x} u^{k} n\right] E^{\tau^{-1 / 4}}\left(f^{k}\right) \cdot \nabla_{n} \varphi \mathrm{d} n \mathrm{~d} x \\
& -\int_{\Omega \times S}(I d+n \otimes n)\left[e_{2} E^{\tau^{-1 / 4}}\left(f^{k}\right)+\nabla_{x} f^{k}\right] \cdot \nabla_{x} \varphi \mathrm{d} n \mathrm{~d} x, \\
& \forall \varphi \in H^{1}(\Omega \times S) .
\end{aligned}
$$

Similarly as the proof of [4], we have

\section{Lemma 2.3}

Let $Z:=\left\{f \in L^{2}(\Omega \times S): f \geq 0\right.$ a.e. $\left.\Omega \times S\right\}$.

If $\left(u^{k-1}, f^{k-1}\right) \in V \times Z$, then there exists $\left(u^{k}, f^{k}\right) \in V \times\left(Z \cap H^{1}(\Omega \times S)\right)$ which solves (15)-(16).

Step 2. Uniform estimate. Suppose that $u_{0} \in H$, $f_{0} \in L^{2}(\Omega \times S)$ and $f_{0} \geq 0$ a.e. on $\Omega \times S$. Let $u^{0}=u^{0}(\tau)$ be the solution of $u^{0}-\tau^{1 / 4} \Delta u^{0}=u^{0}$. Then

$$
\left\|u^{0}\right\|_{L^{2}(\Omega)}^{2}+\tau^{1 / 4}\left\|\nabla_{x} u^{0}\right\|_{L^{2}(\Omega)}^{2} \leq\left\|u_{0}\right\|_{L^{2}(\Omega)}^{2}
$$

and $u^{0} \rightarrow u_{0}$ weakly in $H$ as $\tau \rightarrow 0$. Moreover, let $f^{0}=E^{\tau^{-1 / 4}}\left(f_{0}\right)$. Then $\left(u^{0}, f^{0}\right) \in V \times Z$. Using Lemma 2.3 iteratively, we obtain a sequence of approximate solutions,

$$
\left(u^{k}, f^{k}\right) \in V \times\left(Z \cap H^{1}(\Omega \times S)\right)
$$

to (15)-(16). Similarly as the proof of Lemma 3.5 and Lemma 3.6 in [4], we have

Lemma 2.4

$$
\sup _{k \in N}\left\|f^{k}\right\|_{L^{1}(\Omega \times S)} \leq\left\|f_{0}\right\|_{L^{1}(\Omega \times S)}
$$

For any $k \in N$,

$$
\begin{aligned}
& \frac{1}{2}\left\|u^{k}\right\|_{L^{2}(\Omega)}^{2}+\int_{\Omega \times S} F\left(f^{k}\right) \mathrm{d} n \mathrm{~d} x \\
& +\frac{1}{2} \sum_{i=1}^{k}\left\|u^{k}-u^{k-1}\right\|_{L^{2}(\Omega)}^{2}+\tau \sum_{i=1}^{k}\left\|\nabla_{x} u^{i}\right\|_{L^{2}(\Omega)}^{2} \\
& +2 \tau \sum_{i=1}^{k}\left(\left\|\nabla_{x} \sqrt{f^{i}}\right\|_{L^{2}(\Omega \times S)}^{2}+\left\|\nabla_{n} \sqrt{f^{i}}\right\|_{L^{2}(\Omega \times S)}^{2}\right) \\
& \leq \frac{1}{2}\left\|u_{0}\right\|_{L^{2}(\Omega)}^{2}+\int_{\Omega \times S} F\left(f_{0}\right) \mathrm{d} n \mathrm{~d} x+C\left\|f_{0}\right\|_{L^{1}(\Omega \times S)} .
\end{aligned}
$$


Lemma 2.5 For any $T>0$ we might as well set $N=T / \tau$. Then

$$
\begin{aligned}
& \sup _{1 \leq k \leq N}\left\|f^{k}\right\|_{L^{2}(\Omega \times S)}^{2}+\tau \sum_{k=1}^{N}\left(\left\|\nabla_{x} f^{k}\right\|_{L^{2}(\Omega \times S)}^{2}+\left\|\nabla_{n} f^{k}\right\|_{L^{2}(\Omega \times S)}^{2}\right) \\
& \leq C(T) .
\end{aligned}
$$

Proof. Following the proof of (3.44) in [4], we have that

$$
\begin{aligned}
& \left\|f^{k}\right\|_{L^{2}(\Omega \times S)}^{2}+\tau \sum_{i=1}^{k}\left(\left\|\nabla_{x} f^{i}\right\|_{L^{2}(\Omega \times S)}^{2}+\left\|\nabla_{n} f^{i}\right\|_{L^{2}(\Omega \times S)}^{2}\right) \\
& \leq\left\|f^{0}\right\|_{L^{2}(\Omega \times S)}^{2}+C \tau \sum_{i=1}^{k}\left(\left\|\nabla_{x} u^{i}\right\|_{L^{2}(\Omega)}^{2}+1\right)\left\|f^{i}\right\|_{L^{2}(\Omega \times S)}^{2} .
\end{aligned}
$$

Applying (20), one has $\varepsilon>0$, such that if $\left(u^{0}, f^{0}\right)$ satisfies $\left\|u_{0}\right\|_{L^{2}(\Omega)}^{2}+\int_{\Omega \times S} F\left(f_{0}\right) d n d x \leq \varepsilon$, then $C \tau \sum_{i=1}^{k}\left\|\nabla_{x} u^{i}\right\|_{L^{2}(\Omega)}^{2} \leq \frac{1}{4}$. Furthermore, let $\tau \leq 1 / 4 C$, then

$$
C \tau\left(\left\|\nabla_{x} u^{k}\right\|_{L^{2}(\Omega)}^{2}+1\right)\left\|f^{k}\right\|_{L^{2}(\Omega \times S)}^{2} \leq \frac{1}{2}\left\|f^{k}\right\|_{L^{2}(\Omega \times S)}^{2},
$$

and hence

$$
\begin{aligned}
& \frac{1}{2}\left\|f^{k}\right\|_{L^{2}(\Omega \times S)}^{2} \\
& \leq\left\|f_{0}\right\|_{L^{2}(\Omega \times S)}^{2}+C \tau \sum_{i=1}^{k-1}\left(\left\|\nabla_{\chi} u^{i}\right\|_{L^{2}(\Omega)}^{2}+1\right)\left\|f^{i}\right\|_{L^{2}(\Omega \times S)}^{2} .
\end{aligned}
$$

Using (20) again, and the discrete Gronwall inequality, We finish the proof of (21).

Definition 2.6 Define the piecewise function in $t$ by

$$
u_{\tau}(t, \cdot):=u^{k}(\cdot), \pi_{\tau} u_{\tau}(t, \cdot):=u^{k-1}(\cdot), t \in((k-1) \tau, k \tau]
$$

and the difference quotient of size $\tau$ by

$$
\partial_{t}^{\tau} u_{\tau}(t, \cdot):=\frac{u^{k}(\cdot)-u^{k-1}(\cdot)}{\tau}, t \in((k-1) \tau, k \tau]
$$

Likewise, define $f_{\tau}$ and $\partial_{t}^{\tau} f_{\tau}$.

\section{Lemma 2.7}

$$
\begin{aligned}
& f_{\tau} \geq 0 \text { a.e. on }[0, T] \times \Omega \times S \text {. } \quad \text { (22) ing to (16), we have that for any } \\
& \qquad \begin{array}{l}
\left\|\partial_{t}^{\tau} u_{\tau}\right\|_{L^{2}\left(0, T ; V^{\prime}\right)}=\left(\tau \sum_{k=1}^{N}\left\|\frac{u^{k}-u^{k-1}}{\tau}\right\|_{V^{\prime}}^{2}\right)^{1 / 2} \\
\leq C\left(\tau \sum_{k=1}^{N}\left[\left\|\nabla_{\chi} u^{k}\right\|_{L^{2}(\Omega)}+\left\|u^{k}\right\| u^{k-1}\left\|_{L^{2}(\Omega)}+\right\| f^{k} \|_{L^{2}(\Omega \times S)}\right]^{2}\right)^{1 / 2} \\
\leq C\left(\sum_{k=1}^{N} \int_{(k-1) \tau}^{k \tau}\left[\left\|\nabla_{\chi} u_{\tau}\right\|_{L^{2}(\Omega)}+\left\|u_{\tau}\right\| \pi_{\tau} u_{\tau}\left\|_{L^{2}(\Omega)}+\right\| f_{\tau} \|_{L^{2}(\Omega \times S)}\right]^{2}\right)^{1 / 2} \\
\leq C\left(\left\|\nabla_{\chi} u_{\tau}\right\|_{L^{2}((0, T) \times \Omega)}+\left\|u_{\tau}\right\|_{L^{4}((0, T) \times \Omega)}\left\|\pi_{\tau} u_{\tau}\right\|_{L^{4}((0, T) \times \Omega)}+\left\|f_{\tau}\right\|_{L^{2}((0, T) \times \Omega \times S)}\right) .
\end{array}
\end{aligned}
$$




$$
\begin{aligned}
& \left|\int_{\Omega \times S} \frac{f^{k}-f^{k-1}}{\tau} \cdot \varphi \mathrm{d} n \mathrm{~d} x\right| \\
& \leq \int_{\Omega \times S}\left|u^{k}\right|\left|f^{k}\right|\left|\nabla_{x} \varphi\right| \mathrm{d} n \mathrm{~d} x+\int_{\Omega \times S}\left|\nabla_{n} f^{k}\right|\left|\nabla_{n} \varphi\right| \mathrm{d} n \mathrm{~d} x \\
& +C \int_{\Omega \times S}\left|\nabla_{x} u^{k}\right|\left|f^{k}\right|\left|\nabla_{n} \varphi\right| \mathrm{d} n \mathrm{~d} x \\
& +C \int_{\Omega \times S}\left(\left|\nabla_{x} f^{k}\right|+\left|f^{k}\right|\right)\left|\nabla_{x} \varphi\right| \mathrm{d} n \mathrm{~d} x .
\end{aligned}
$$

Consequently

$$
\begin{aligned}
& \left\|\frac{f^{k}-f^{k-1}}{\tau}\right\|_{\left(H^{3}(\Omega \times S)\right)^{\prime}} \leq C\left(\left\|u^{k}\right\|_{H^{1}(\Omega)}\left\|f^{k}\right\|_{L^{2}(\Omega \times S)}+\left\|f^{k}\right\|_{H^{1}(\Omega \times S)}\right) .
\end{aligned}
$$

Similarly as the proof of (26), we have from (23) and (24) that $\left\|\partial_{t}^{\tau} f_{\tau}\right\|_{L^{2}\left(0, T ;\left(H^{3}(\Omega \times S)\right)^{\prime}\right)^{\prime}}^{2} \leq C(T)$.

Step 3. Convergence. With the above uniform estimates at hand, we can use the Aubin-Lions lemma for time-piecewise functions (see [9]) to perform the compactness argument. This concludes the proof of Theorem 2.1 .

\section{REFERENCES}

[1] M. Doi and S. F. Edwards, "The Theory of Polymer Dynamics," Oxford University Press, Oxford, 1986.

[2] F. Otto and A. E. Tzavaras, "Continuity of Velocity Gradient in Suspensions of Rod-Like Molecules," Communications in Mathematical Physics, Vol. 277, No. 3, 2008, pp. 729-758.

http://dx.doi.org/10.1007/s00220-007-0373-5

[3] C. Helzel, F. Otto and A. E. Tzavaras, "A Kinetic Model for the Sedimentation of Rod-Like Particles," 2011.

[4] X. Q. Chen, X. L. Li and J.-G. Liu, "Existence and Uniqueness of Global Weak Solution to a Kinetic Model for the Sedimentation of Rod-Like Particles," Communications in Mathematical Sciences, 2013.

[5] P. Constantin, "Nonlinear Fokker-Planck Navier-Stokes Systems," Communications in Mathematical Sciences, Vol. 3, No. 4, 2005, pp. 531-544. http://dx.doi.org/10.4310/CMS.2005.v3.n4.a4

[6] P. L. Lions and N. Masmoudi, "Global Solutions of Weak Solutions to Some Micro-Macro Models," Comptes Rendus Mathematique, Vol. 345, No. 1, 2007, pp. 15-20. http://dx.doi.org/10.1016/j.crma.2007.05.011

[7] Y. Z. Sun and Z. F. Zhang, "Global Well-Posedness for the 2D Micro-Macro Models in the Bounded Domain," Communications in Mathematical Physics, Vol. 303, No. 2, 2011, pp. 361-383. http://dx.doi.org/10.1007/s00220-010-1170-0

[8] W. J. Barrett and E. Süli, "Existence and Equilibration of Global Weak Solutions to Kinetic Models for Dilute Polymers I: Finitely Extensible Nonlinear Bead-Spring Chains," Mathematical Models and Methods in Applied Sciences, Vol. 21, No. 6, 2011, pp. 1211-1289. http://dx.doi.org/10.1142/S0218202511005313

[9] M. Dreher and A. Jüngel, "Compact Families of Piecewise Constant Funcitons in $\mathrm{L}^{\wedge} \mathrm{p}(0, \mathrm{~T} ; \mathrm{B})$," Nonlinear Analysis, Vol. 75, No. 6, 2012, pp. 3072-3077. http://dx.doi.org/10.1016/j.na.2011.12.004 